\title{
A RANK STATISTIC FOR NON-PARAMETRIC $K$-SAMPLE AND CHANGE POINT PROBLEMS
}

\author{
Yoichi Nishiyama*
}

\begin{abstract}
We consider $k$-sample and change point problems for independent data in a unified way. We propose a test statistic based on the rank statisitcs. The asymptotic distribution under the null hypothesis is shown to be the supremum of the 2-dimensional standard Brownian pillow. Also, the test is shown to be consistent under the alternative that $k$ distribution functions are linearly independent. It is important from practical point of view that our test is not only asymptotically distribution free but also distribution free even for fixed finite sample.
\end{abstract}

Key words and phrases: Empirical process, invariance principle, rank statistic, weak convergence.

\section{Introduction}

This paper studies the $k$-sample and change point problems in a unified way. Both problems have long histories.

Kiefer (1959) considered $k$-sample Kolmogorov-Smirnov and Cramér-von Mises tests, while Scholz and Stephens (1987) studied the $k$-sample AndersonDarling test. The approaches based on the empirical distribution functions are naive, but the limit distributions are often difficult to compute. On the other hand, other approaches often need some restrictions on alternatives. For example, Jonckheere (1954) considered the $k$-sample test against ordered alternatives (see also Odeh (1971)), while Mack and Wolfe (1981) treated that against umbrella alternatives. Hettmansperger and Norton (1987) considered the $k$-sample problem against a patterned alternative. (On the contrary, the alternative in our approach just requires that the $k$ distribution functions are linearly independent.)

Regarding the change point problems, many authors have considered parametric and non-parametric approaches. See, e.g., the book by Csörgö and Horváth (1997). Since we are interested in a non-parametric approach, we only review preceding results in that direction. Pettitt (1979) applied the WilcoxonMann-Whitney statistic to the non-parametric change point problem. Lombard (1987) proposed a procedure based on quadratic form rank statistics to test one or more change points. (One of the interesting points of our approach is that we do not require prior knowledge about how many change points exist under the alternative.) Recently, based on Lombard's approach, Murakami (2010) introduced a rank statistic for the change point problem of location-scale parameters. Praagman (1988) established the Bahadur efficiency of some rank tests for the non-parametric change point problem. As for estimation problems, Carlstein

\footnotetext{
Received January 4, 2011. Revised March 24, 2011. Accepted April 15, 2011.

*The Institute of Statistical Mathematics, 10-3 Midori-cho, Tachikawa, Tokyo 190-8562, Japan.
} 
(1988) proposed an estimator for a change point without assuming any specific structure of the underlying distribution, among others.

Our idea comes from a kind of CUSUM empirical process, although the resulting test is a rank statistic. Our test has the following merits. (1) It is distribution free under the null hypothsis; the distribution of our test depends only on the total sample size. (2) The asymptotic distribution under the null hypothesis is the supremum of the absolute value of the 2-dimensional standard Brownian pillow; of course, it does not depend on $k$. (3) Our alternative is natural; we only assume that the $k$ distribution functions are linearly independent.

(4) Our test is easy to compute.

The organization of the rest of this paper is as follows. In Section 2, we state some asymptotic results under the null and alternative hypotheses. These results are proved in Section 3. In Section 4, we present some simulation studies.

\section{Asymptotic results}

Let us describe two problems which we consider in this paper.

The first problem is the so-called $k$-sample problem. Let $X_{1}^{c}, \ldots, X_{n_{c}}^{c}, c=$ $1, \ldots, k$, be 1 -dimensional independent data such that for every $c=1, \ldots, k$ the data $X_{1}^{c}, \ldots, X_{n_{c}}^{c}$ come from a 1-dimensional continuous distribution $F^{c}$. We wish to test the hypotheses:

$H_{0}: F^{c}$ 's are the same for all $c=1, \ldots, k$ (we denote the common distribution by $F)$;

$H_{1}: F^{c}$ 's are distribution functions that are linearly independent: that is, for weight constants $\left(w_{1}, \ldots, w_{k}\right)$ such that $\sum_{c} w_{c}=0$, it holds that $\sum_{c} w_{c} F^{c}(\cdot) \equiv 0$ imply $w_{c}=0$ for all $c$. (The additional constraint " $\sum_{c} w_{c}=0$ " is not a real restriction for the linear independence because of the fact that $F^{c}(\infty)=1$ for all c.)

The second problem is the so-called change point problem. Let $X_{1}, \ldots, X_{n}$ be 1-dimensional independent data. We wish to test the hypothesis:

$H_{0}$ : all $X_{i}$ 's come from a certain continuous distribution $F$;

$H_{1}$ : there exist $0=u_{0}<u_{1}<\cdots<u_{k}=1$ such that $X_{i}, i=\left[u_{c-1} n\right]+$ $1, \ldots,\left[u_{c} n\right], c=1, \ldots, k$, come from a distribution $F^{c}$ where $F^{c}$ 's are functions that are linearly independent.

We can treat the second problem as a special case of the first, by regarding $n_{c}=\left[u_{c} n\right]-\left[u_{c-1} n\right]$, hence from now on we deal with the first problem. We shall assume that $\gamma_{c}=\lim \frac{n_{c}}{n}$, where $n=\sum_{c} n_{c}$, as $n_{c} \rightarrow \infty$ for every $c=1, \ldots, k$. (We assume that at least two $\gamma_{c}$ 's are positive.) This is a natural assumption for the second problem where $\gamma_{c}=u_{c}-u_{c-1}$, and throughout this paper we consider this asymptotic scheme.

In the first problem, let us set

$$
\left\{X_{1}, \ldots, X_{n}\right\}=\left\{X_{1}^{1}, \ldots, X_{n_{1}}^{1}, \ldots, X_{1}^{k}, \ldots, X_{n_{k}}^{k}\right\}
$$

Let us denote by $\left\{X_{(1)}, \ldots, X_{(n)}\right\}$ the order statistics and by $\left\{R_{1}, \ldots, R_{n}\right\}$ the 
rank statistics of the data $\left\{X_{1}, \ldots, X_{n}\right\}$. We propose the test statistic

$$
D_{n}=\frac{1}{\sqrt{n}} \max _{1 \leq i, j \leq n}\left|\sum_{q=1}^{i} 1\left\{R_{q} \leq j\right\}-\frac{i j}{n}\right| \text {. }
$$

The main result of this paper is the following.

THEOREM 1. (i) Under the null hypothesis $H_{0}$, it holds that, as $n \rightarrow \infty$,

$$
D_{n} \stackrel{d}{\rightarrow} \sup _{0 \leq s, t \leq 1}\left|W^{\circ}(s, t)\right|,
$$

where $W^{\circ}$ is a centered Gaussian process with the covariance

$$
\mathrm{E}\left[W^{\circ}\left(s_{1}, t_{1}\right) W^{\circ}\left(s_{2}, t_{2}\right)\right]=\left(s_{1} \wedge s_{2}-s_{1} s_{2}\right)\left(t_{1} \wedge t_{2}-t_{1} t_{2}\right) .
$$

Hence the test is asymptotically distribution free under $H_{0}$.

(ii) Under the alternative $H_{1}$, it holds that, as $n_{c} \rightarrow \infty$ for every $c=1, \ldots, k$,

$$
D_{n} \geq \sqrt{n} \max _{1 \leq c_{*} \leq k} \sup _{x} B^{c_{*}}(x)-o(\sqrt{n})-O_{P}(1),
$$

where

$$
B^{c_{*}}(x)=\left|\sum_{c \leq c_{*}} \gamma_{c}\left(1-u_{*}\right) F^{c}(x)-\sum_{c>c_{*}} \gamma_{c} u_{*} F^{c}(x)\right|
$$

and where $u_{*}=\sum_{c \leq c_{*}} \gamma_{c}$. (Notice that the sum of coefficients of $F^{c}$ 's is zero, so by assumption $\sup _{x} B^{c_{*}}(x)$ is positive.) Hence the test is consistent under $H_{1}$.

It is important from a practical point of view that we do not assume any specific structure on the distributions $F^{c}$ s. Our approach is purely a non-parametric one.

It is also important to notice that the value $k$ is not used in the construction of our test, so we can treat it as an unknown parameter. Compare our way of constructing the test statistic with the ones for other tests in the context of the change point problems where the value $k$ has often been assumed to be known.

\section{Proof of Theorem 1}

To begin with, let us notice that

$$
\begin{aligned}
\tilde{D}_{n} & \left.:=\sup _{u \in[0,1]} \sup _{x \in \mathbb{R}} \mid \frac{1}{\sqrt{n}} \sum_{i=1}^{n}(1\{i \leq u n\}-u\}\right) 1\left\{X_{i} \leq x\right\} \mid \\
& =\max _{1 \leq i \leq n} \sup _{x \in \mathbb{R}} \frac{1}{\sqrt{n}}\left|\sum_{q=1}^{i} 1\left\{X_{q} \leq x\right\}-\frac{i}{n} \sum_{q=1}^{n} 1\left\{X_{q} \leq x\right\}\right|+O\left(\frac{1}{\sqrt{n}}\right) \\
& =\max _{1 \leq i, j \leq n} \frac{1}{\sqrt{n}}\left|\sum_{q=1}^{i} 1\left\{X_{q} \leq X_{(j)}\right\}-\frac{i}{n} \sum_{q=1}^{n} 1\left\{X_{q} \leq X_{(j)}\right\}\right|+O\left(\frac{1}{\sqrt{n}}\right) \\
& =D_{n}+O\left(\frac{1}{\sqrt{n}}\right) .
\end{aligned}
$$




\section{HO (no change point)}

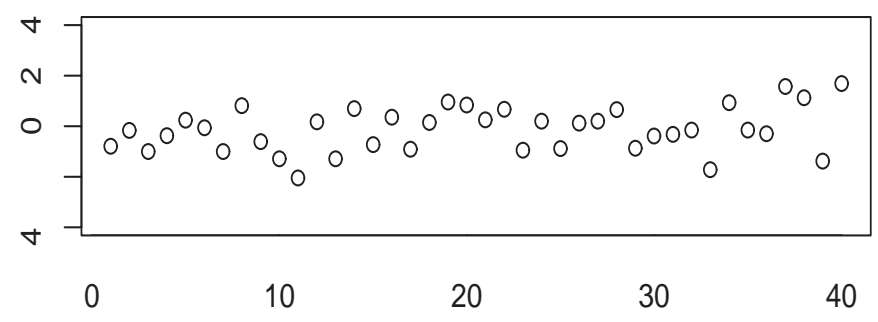

H1 (a change point)

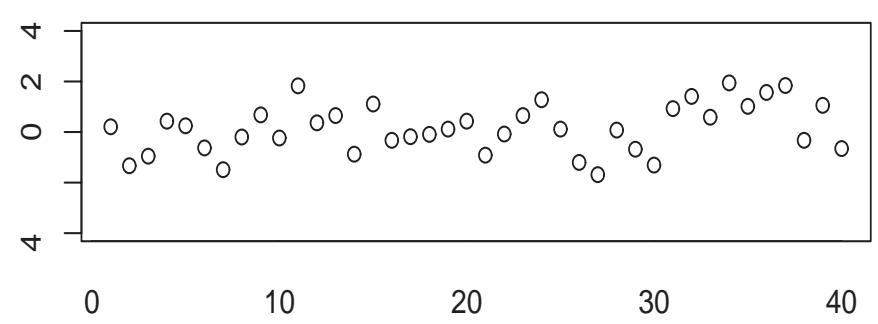

Figure 1. Plots of the data.

Proof of Theorem 1 (i). Since $\sup _{u \in[0,1]}\left|\frac{1}{n} \sum_{i=1}^{n}(1\{i \leq u n\}-u)\right| \leq \frac{1}{n}$, the random variable $\tilde{D}_{n}$ is asymptotically equivalent to the supremum of the absolute value of

$$
u, x \leadsto \frac{1}{\sqrt{n}} \sum_{i=1}^{n}(1\{i \leq n u\}-u)\left(1\left\{X_{i} \leq x\right\}-F(x)\right),
$$

which converges weakly in $\ell^{\infty}([0,1] \times \mathbb{R})$ to the centered Gaussian process $u, x \leadsto$ $W^{\circ}\left(u, F(x)\right.$ ). (We denote by $\ell^{\infty}(T)$ the space of bounded functions on $T$, and equip it with the uniform metric. See e.g. van der Vaart and Wellner (1996) for the weak convergence theory in this space.) Hence the result follows from the continuous mapping theorem.

Proof of Theorem 1 (ii). Let $c_{*}$ be any of the index $c$. We also denote by $x_{*}$ the $\operatorname{argusup}$ of $x \mapsto B^{c_{*}}(x)$. Notice that

$$
\begin{aligned}
\tilde{D}_{n} \geq & \frac{1}{\sqrt{n}}\left|\sum_{i=1}^{n}\left(1\left\{i \leq n u_{*}\right\}-u_{*}\right) F^{\tau(i)}\left(x_{*}\right)\right| \\
& -\left|\frac{1}{\sqrt{n}} \sum_{i=1}^{n}\left(1\left\{i \leq n u_{*}\right\}-u_{*}\right)\left(1\left\{X_{i} \leq x_{*}\right\}-F^{\tau(i)}\left(x_{*}\right)\right)\right|,
\end{aligned}
$$




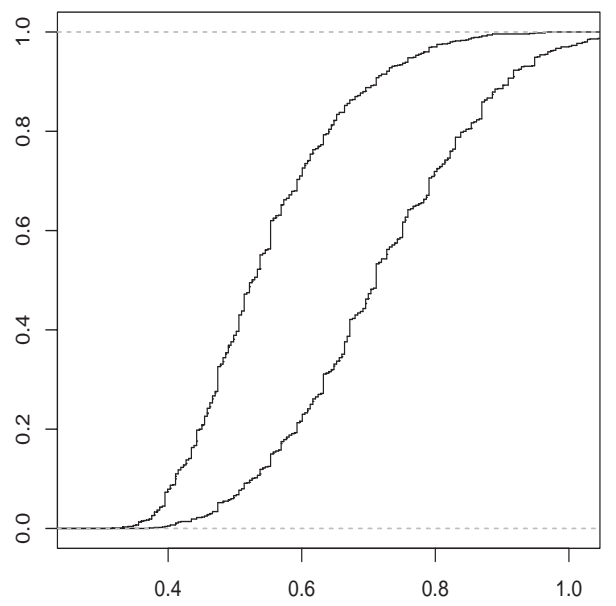

Figure 2. EDF of 1000 simulations of $D_{40}$ under $H_{0}$ (left) and $H_{1}$ (right).

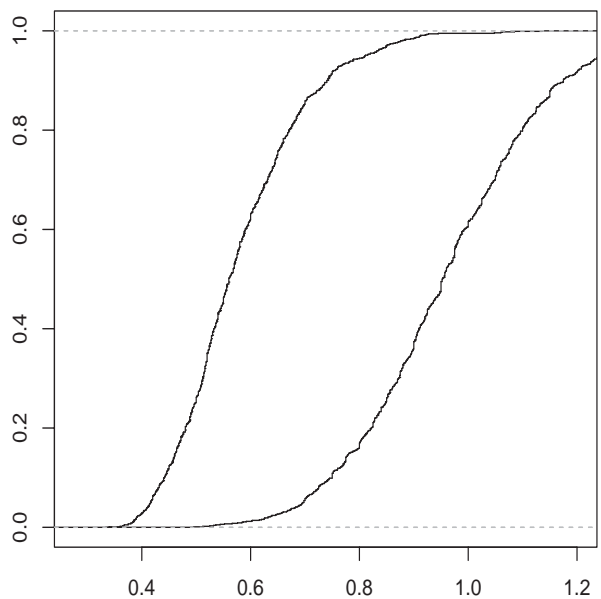

Figure 3. EDF of 1000 simulations of $D_{100}$ under $H_{0}$ (left) and $H_{1}$ (right).

where $\tau(i)=c$ for $i=n_{c-1}+1, \ldots, n_{c}$. By the central limit theorem, the second term on the right hand side converges weakly to a tight limit. The first term can be written as $\sqrt{n}\left(B^{c_{*}}\left(x_{*}\right)+o(1)\right)$. The proof is finished.

\section{Remark for non-asymptotic case}

The way of defining the test statistic (2.1) has a merit not only for asymptotic study but also for the finite simple argument. Since the test is defined only through the rank statistics, it is distribution free under $H_{0}$ as far as the underlying distribution $F$ is a 1-dimensional continuous distribution. So, we can compute the p-values for fixed $n$ by computer simulation, by setting the underlying distribution $F$ to be, for example, the uniform distribution. The p-values 


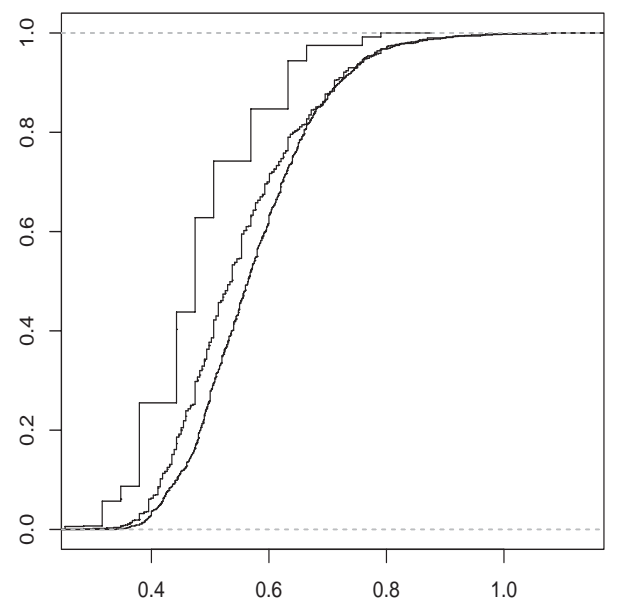

Figure 4. EDF of 1000 simulations of $D_{10}, D_{40}$ and $D_{100}$ under $H_{0}$.

obtained by Monte-Carlo simulation are much better than the one obtained by using the asymptotic distribution $\sup _{s, t}\left|W^{\circ}(s, t)\right|$, especially when $n$ is not very large.

Here we present some numerical results by figures. For illustration, we demonstrate the simulation for the change point problem with $k=2$. Figure 1 is the plots of the independent data $X_{1}, \ldots, X_{n}$ from the Gaussian distribution $N(0,1)$ [upper], and $X_{1}, \ldots, X_{[3 n / 4]}$ from $N(0,1)$ and $X_{[3 n / 4]+1}, \ldots, X_{n}$ from $N(1,1)$ [lower] (with $n=40)$.

We perform 1000 simulations for the computation of the test statistic $D_{n}$. Figure $2(n=40)$ and Figure $3(n=100)$ present the empirical distribution functions (EDF) under $H_{0}$ and $H_{1}$.

Finally, we draw the empirical distribution functions of 1000 simulations for $D_{n}$ under $H_{0}$ for $n=10,40,100$ (Figure 4 ). We set $F$ to be the uniform distribution, but the choice of the underlying distribution is not important because we know that the test is distribution free. So we can compute the approximate p-values even for small $n$ based on this kind of computer simulation.

\section{Acknowledgements}

This work was supported by Grant-in-Aid for Scientific Research (C), 21540157, from Japan Society for the Promotion of Science.

\section{REFERENCES}

Carlstein, E. (1988). Nonparametric change-point estimation, Ann. Statist., 16, 188-197.

Csörgö, M. and Horváth, L. (1997). Limit Theorems in Change-Point Analysis, Wiley, New York.

Hettmansperger, T. P. and Norton, R. M. (1987). Test for patterned alternatives in $k$-sample problems, J. Amer. Statist. Assoc., 82, 292-299.

Jonckheere, A. R. (1954). A distribution free $k$-sample test against ordered alternatives, Biometrika, 41, 133-145. 
Kiefer, J. (1959). K-sample analogues of the Kolmogorov-Smirnov and Cramér-V. Mises tests, Ann. Math. Statist., 30, 420-447.

Lombard, F. (1987). Rank tests for changepoint problems, Biometrika, 74, 615-624.

Mack, G. A. and Wolfe, D. A. (1981). $K$-sample rank tests for umbrella alternatives, J. Amer. Statist. Assoc., 76, 175-181.

Murakami, H. (2010). A rank statistic for the change-point problem and its application, J. Jpn. Soc. Comp. Statist., 23, 27-40.

Odeh, R. E. (1971). On Jonckheere's $k$-sample test against ordered alternatives, Technometrics, 13, 912-918.

Pettitt, A. N. (1979). A non-parametric approach to the change-point problem, Appl. Statist., 28, 126-135.

Praagman, J. (1988). Bahadur efficiency of rank tests for the change-point problem, Ann. Statist., 16, 198-217.

Scholz, F. W. and Stephens, M. A. (1987). K-sample Anderson-Darling tests, J. Amer. Statist. Assoc., 82, 918-924.

van der Vaart, A. W. and Wellner, J. A. (1996). Weak Convergence and Empirical Processes: With Applications to Statistics, Springer-Verlag, New York. 